\title{
Correction to: Intrauterine resuscitation during the second stage of term labour by maternal hyperoxygenation versus conventional care: study protocol for a randomised controlled trial (INTEREST O2)
}

Lauren M. Bullens ${ }^{1,2^{*}}$, Alexandra D. J. Hulsenboom ${ }^{1}$, Suzanne Moors ${ }^{1}$, Rohan Joshi3 ${ }^{3,4}$, Pieter J. van Runnard Heimel', M. Beatrijs van der Hout-van der Jagt ${ }^{1,2}$, Edwin R. van den Heuvel ${ }^{5}$ and S. Guid Oei ${ }^{1,2}$

\section{Correction}

Following publication of the original article [1], the authors noticed that the sample size for the study group was incorrectly reported in the Methods section. The correct number of patients to participate in the study is 116 .

\section{Author details}

'Department of Obstetrics and Gynaecology, Máxima Medical Centre, PO Box 7777, 5500 MB Veldhoven, The Netherlands. ${ }^{2}$ Department of Electrical Engineering, Eindhoven University of Technology, PO Box 513, 5500 MB Eindhoven, The Netherlands. ${ }^{3}$ Department of Clinical Physics, Máxima Medical Centre, PO Box 7777, 5500 MB Veldhoven, The Netherlands. ${ }^{4}$ Department of Industrial Design, Eindhoven University of Technology, PO Box 513, 5500 MB Eindhoven, The Netherlands. ${ }^{5}$ Department of Mathematics and Computer Science, Eindhoven University of Technology, PO Box 513,

5500 MB Eindhoven, The Netherlands.

Received: 4 October 2018 Accepted: 4 October 2018

Published online: 23 October 2018

\section{Reference}

1. Bullens $L M$, et al. Intrauterine resuscitation during the second stage of term labour by maternal hyperoxygenation versus conventional care: study protocol for a randomised controlled trial (INTEREST O2). Trials. 2018;19:195. https://doi.org/10.1186/s13063-018-2567-x.

\footnotetext{
*Correspondence: laurenbullens@gmail.com

'Department of Obstetrics and Gynaecology, Máxima Medical Centre, PO Box 7777, 5500 MB Veldhoven, The Netherlands

${ }^{2}$ Department of Electrical Engineering, Eindhoven University of Technology, PO Box 513, 5500 MB Eindhoven, The Netherlands
} 